\title{
Oportunidades e desafios no desenvolvimento de Recursos Educacionais Abertos Multiculturais
}

\author{
Edie Correia Santana, Ismar Frango Silveira
}

Programa de Pós-Graduação em Engenharia Elétrica e Computação - Universidade

Presbiteriana Mackenzie (UPM) - São Paulo - SP - Brasil

edie.santana@svc.ifmt.edu.br, ismar.silveira@mackenzie.br

\begin{abstract}
The observance of multicultural aspects in open educational resources design is a very important issue, since it impacts directly on the ability of students to have access to it, as well as to learn from its content, since some could not fit to some culture, while are adequate to other. The main goal of this paper is to point out the relevance of this subject as a challenge for Computers and Education field, thus contributing to a new perspective in the context of the design of multicultural open educational resources.
\end{abstract}

Resumo. A atenção aos aspectos multiculturais no projeto de recursos educacionais abertos se demonstra de alta importância, pois pode impactar na capacidade do aluno acessar o recurso e aprender a partir de seu conteúdo, já que determinadas representações, apropriadas para uma cultura, podem não ser adequadas para outra. Assim, o objetivo deste trabalho é delinear a relevância desse tema como um desafio da área de Informática na Educação, contribuindo com novas perspectivas no design de recursos educacionais abertos multiculturais.

\section{Introdução}

Vivemos em uma sociedade multicultural em que as relações entre as pessoas estão em crescente transformação, majoritariamente impulsionadas, ou ao menos afetadas, pela evolução e onipresença das tecnologias da informação e da comunicação (TIC). O amplo acesso à informação e às diversas formas de comunicação tem criado oportunidades para compartilhamento de informações e trocas culturais, que sem as TIC dificilmente seriam possíveis.

No contexto educacional, novas oportunidades de trocas culturais também têm impactado a maneira como se produz e consome conhecimento. A Web 2.0 (e suas renumerações subsequentes) e o advento das redes sociais reverberam na educação demandas por espaços de aprendizagem mais flexíveis e com participação ativa de todos os agentes educacionais, exigindo o repensar das práticas de ensino e dos modelos pedagógicos. Nessa direção, o Desafio 4 da SBC (2016), intitulado "Acesso Participativo e Universal do Cidadão Brasileiro ao Conhecimento" destaca que "existem barreiras tecnológicas, educacionais, culturais, sociais e econômicas, que impedem o acesso e a interação", prevendo a necessidade de se buscar "a extensão de sistemas computacionais ao cidadão comum, em sua diversidade, respeitando suas diferenças".

Nesse contexto, o recente movimento de Educação Aberta ganha impulso sobretudo porque a essência de sua filosofia é quebrar as barreiras que limitam o acesso 
à educação, proporcionando maiores oportunidades de aprendizagem por meio do amplo acesso, independente de local, cultura e contexto. (OKADA e OKADA, 2007).

Os conceitos básicos desse movimento apontam para o uso efetivo de Recursos Educacionais Abertos (REA), o que potencializa tanto o acesso quanto as possibilidades de reúso, visto que ditos recursos deveriam poder ser alterados, combinados e adaptados para diferentes contextos. Assim, REA, quando projetados de maneira a se adequar aos princípios de abertura, podem satisfazer a diferentes necessidades advindas de outras culturas, costumes, crenças e valores. Porém, é temerária a ideia de se oferecer recursos educacionais, como livros didáticos e recursos multimídia, neutros quanto aos elementos culturais ou até mesmo sem esses elementos, visto que a cultura é parte essencial de qualquer processo pedagógico - de forma que se torna impossível remover os traços culturais de um recurso educacional sem desfigurar o seu próprio papel de elemento de aprendizagem. Fato é que os elementos culturais estão sempre presentes e podem oferecer barreiras, muitas vezes intransponíveis, ao usuário final. Reconhecer a existência desse tipo de barreira é um passo importante em direção a uma educação mais aberta e inclusiva (AMIEL et al., 2011).

Todo este cenário representa um importante desafio para a área de Informática na Educação, que será detalhado nas seções deste artigo. O presente trabalho considera que o conceito de abertura deve ser ampliado para além da socialização de recursos, materiais e conteúdos educacionais. Deve-se inserir o conceito de abertura também no design de REA, chegando a algo que pode ser útil não só para a criação de REA com possibilidades de reutilização do material, mas também para pensar em adaptação demandada por demandas culturais como diversidades linguísticas, classe social, sexualidade, habilidades, entre outros aspectos.

\section{Recursos Educacionais Abertos e Multiculturalidade}

A UNESCO (2002) definiu o termo Recursos Educacionais Abertos referindo-se a materiais de ensino, aprendizagem e pesquisa em qualquer suporte ou mídia, que estão sob domínio público ou estão licenciados de maneira aberta, permitindo que sejam utilizados ou adaptados por terceiros. De acordo com tal definição, REA podem ser desde livros didáticos e artigos acadêmicos até notas de aulas e cursos, além de software, vídeos, e outros artefatos que possam apoiar a aprendizagem e o acesso ao conhecimento.

$\mathrm{Na}$ visão de Banzato (2012), os REAs se baseiam em três princípios fundamentais: os materiais devem ter valor educacional; um recurso só é considerado REA se for totalmente aberto, sem custos ou qualquer restrição, estando disponível para reutilização, revisão, recontextualização e redistribuição; e as tecnologias devem ser capazes de dar suporte ao desenvolvimento e às questões pedagógicas dos REAs. Wiley (2014) vem a sistematizar tais fundamentos em seus já conhecidos "Princípios 5R de abertura", a saber: Reúso (o direito de usar o recurso em distintos contextos); Revisão (o direito de adaptar, ajustar, modificar ou alterar o recurso); Remixagem (no que se refere à combinação de diferentes recursos para a criação de um novo); Redistribuição (de cópias do conteúdo original ou alterado); e Retenção (da licença e dos direitos de manter, possuir e controlar as cópias dos recursos) - este último princípio adicionado a uma lista anterior proposta pelo mesmo autor, com originalmente quatro princípios. 
No debate referente à reutilização e adaptação de recursos em um ambiente educacional, é parte imprescindível, na visão de Gasparini (2013), o contexto cultural do aluno que é expresso pelos aspectos e background culturais do mesmo. Apesar de não haver consenso na definição de cultura, este termo pode ser entendido em um sentido mais amplo como um conjunto de parâmetros que podem ser utilizados para diferenciar a coletividade social e, na visão de D'Ambrosio (1999), esses parâmetros podem ser normas de comportamento, conhecimento, valores morais, estilos, mitos, entre outros. Em cada cultura, portanto, existe um modo de falar, de pensar, de agir e de se relacionar com outros indivíduos - de fato, vivemos em uma sociedade na qual podemos perceber diversos grupos sociais com suas respectivas culturas e práticas. Nesse sentido, o termo multiculturalismo vem tendo destaque em discussões sobre esse contexto, pois corrobora com a perspectiva de percebermos que vivemos numa sociedade com diversas culturas. Canen (2008), então, diz que o "multiculturalismo é definido como um conjunto de princípios e práticas voltadas para valorização da diversidade cultural e para o desafio de preconceitos e estereótipos a ela relacionado".

Portanto, em se tratando do uso de recursos educacionais, o contexto cultural inclui a experiência e formação cultural do aluno e pode ter um grande impacto em sua capacidade e eficiência para aprender um determinado conteúdo, já que contexto cultural se refere a diferentes aspectos, tais como os aspectos social, ideológico, político, étnico, as diferentes línguas, valores, normas e as questões relacionados ao gênero (CHANDRAMOULI et al., 2008).

Para Gasparini (2013), o contexto cultural de um usuário molda sua percepção sobre características do sistema: um determinado contexto cultural possivelmente faz com que um usuário se concentre em um conjunto de informações e ignore outros. Assim, as características de um artefato computacional apropriadas para uma cultura podem não ser adequadas para outra e o projeto deste artefato precisa ser adaptado para diferentes culturas.

\section{Trabalhos relacionados}

Na pesquisa acerca de REA multiculturais, alguns autores têm defendido a ideia que existem fatores que podem inibir ou prejudicar o acesso, ou ainda a apresentação de conteúdos aos alunos. Outros, sabendo desses fatores, têm procurado estratégias para representar soluções que podem dar suporte a múltiplas culturas em REA. A seguir são apresentados alguns trabalhos que apresentam pesquisas nessa direção.

Em um relatório, a OCDE (Organização para a Cooperação e Desenvolvimento Econômico) salientou que as barreiras à utilização ou à produção de REA podem ser categorizadas em barreiras técnicas, econômicas, sociais, políticas e jurídicas (OCDE, 2007). Com um maior nível de detalhamento, Hatakka (2009) identifica 11 fatores que podem inibir o uso efetivo de REA; dentre esses fatores a autora inclui regras e restrições educacionais, linguagem utilizada, relevância, acesso, recursos técnicos, aspectos qualidade, propriedade intelectual, percepção, conhecimento em informática, capacidade de ensino, bem como determinadas práticas e tradições de ensino.

O relatório técnico do GranDIHC-BR (Baranauskas et al., 2012) também aponta nessa direção e amplia a discussão para as frentes de acessibilidade e responsividade, ao incluir aspectos de acessibilidade para pessoas com distintas deficiências, físicas e 
cognitivas, e também por envolver a questão de múltiplos dispositivos de acesso. $\mathrm{O}$ referido documento, em seu Desafio 2, indica que "é necessária a construção de sistemas que possam ser generalizados para múltiplos dispositivos e ao mesmo tempo especializados para os diferentes usuários com diferentes necessidades". Portanto, é essencial considerarmos essas questões ao se desenvolver REA para que esses sejam relevantes, úteis e acessíveis a professores e estudantes.

As atuais discussões sobre multiculturalidade em recursos digitais para a aprendizagem são fruto do amadurecimento das pesquisas relacionadas à terminologia de Objetos de Aprendizagem (OA), em cujo contexto vários trabalhos apontam que as questões culturais merecem destaque e atenção (CHEN, 2010; HATAKKA, 2009; AMIEL et al., 2010; MUÑOZ-ARTEAGA et al., 2011).

Chen (2010) sustenta que a relevância de um recurso educacional é sustentada pela sua flexibilidade, pela possibilidade que ele apresenta de adaptação ao contexto local do estudante e pela possibilidade de reutilização. A adaptação ao contexto do estudante, diz respeito a várias perspectivas, entre elas a cultural, por exemplo, alguns exemplos dados em países desenvolvidos podem não ser relevantes para estudantes oriundos de outras culturas e/ou de países menos desenvolvidos.

Amiel et al. (2009) apresentam razões e estratégias para que questões culturais sejam elencadas no processo de design de recursos educacionais, no caso, objetos de aprendizagem. Em outro trabalho, Amiel et al. (2011) apresentam uma proposta em que a arquitetura de cada REA variará dependendo da abordagem de cultura escolhida. Para isso, os autores propõem quatro classes para diferenciar abordagens de culturalização no contexto de recursos educacionais, sendo elas: RE (Recursos Educacionais), RESC (Recursos Educacionais com Saliências Culturais), n-Cultura e REAC (Recursos Educacional com Adaptações Culturais)..

Já Muñoz-Arteaga et al. (2011) propõem o desenvolvimento de um REA baseado no padrão de projeto Presentation-Abstraction-Control (PAC). Nesse trabalho, diferentes aspectos culturais possíveis do conteúdo do recurso são representados por agentes PAC que poderiam ser selecionados pelos de acordo as necessidades de aprendizagem dos alunos.

Em síntese, tem-se que muitas das pesquisas relacionadas à REA multiculturais se concentraram no reconhecimento e proposição de barreiras e dificuldades que prejudicam o acesso do aluno ao conhecimento por meio de REA. As abordagens de Amiel et al. (2011) e Muñoz-Arteaga et al. (2011) se diferenciaram das outras por apresentarem propostas mais relacionadas com projeto e arquitetura de REA, mas ainda falta uma tratativa mais sistêmica, ou seja, é necessário considerar as questões culturais não somente em algumas partes ou fases do processo de design de REA, mas no processo de design como um todo.

\section{Oportunidades e desafios}

Com base no contexto anteriormente detalhado e na análise de trabalhos correlatos, identificam-se na tabela a seguir um conjunto de questões e oportunidades que devem ser consideradas na busca por soluções para este desafio, bem como barreiras na execução dessas questões e os possíveis benefícios a serem alcançados. Na sequência, 
apresentam-se detalhes sobre os itens mostrados na Tabela 1 e também um conjunto de ações para medir o sucesso de cada ação e quais procedimentos a serem tomados para enfrentar esse desafio na década vindoura.

Tabela 1: Oportunidades, barreiras e benefícios

\begin{tabular}{|c|c|c|}
\hline Oportunidades & Barreiras & Benefícios \\
\hline $\begin{array}{l}\text { Definição de } \\
\text { processos de } \\
\text { design } \\
\text { instrucional }\end{array}$ & $\begin{array}{l}\text { Um projeto instrucional resolve } \\
\text { uma classe de demandas } \\
\text { educacionais. No contexto atual } \\
\text { existem vários tipos de demandas. } \\
\text { Envolvimento das partes } \\
\text { interessadas no processo de design. } \\
\text { Poucas iniciativas de propostas para } \\
\text { design de REA que levem em } \\
\text { consideração a complexidade desse } \\
\text { recurso. }\end{array}$ & $\begin{array}{l}\text { Iniciativas de novos modelos de } \\
\text { processos beneficiariam alunos, } \\
\text { professores e instituições com suas } \\
\text { diversas demandas educacionais. } \\
\text { Processos de design adaptáveis } \\
\text { seriam muito interessantes para } \\
\text { resolver os problemas oriundos das } \\
\text { informações contextuais dos alunos. } \\
\text { Considerar as partes envolvidas no } \\
\text { processo de design resulta em } \\
\text { recursos que fazem mais sentido às } \\
\text { pessoas. } \\
\text { Considerar que um REA é um } \\
\text { artefato complexo, assim como um } \\
\text { software, mas não o considerar } \\
\text { como um software e não utilizar } \\
\text { modelos da engenharia, pode fazer } \\
\text { com o que o REA seja mais efetivo. }\end{array}$ \\
\hline $\begin{array}{l}\text { Evidências de } \\
\text { reúso }\end{array}$ & $\begin{array}{l}\text { Há pouca discussão quanto a como } \\
\text { esse o reúso pode acontecer. O } \\
\text { reúso existe e é possível, porém, } \\
\text { não é trivial. } \\
\text { Falta a percepcção por parte dos } \\
\text { usuários e professores a cerca da } \\
\text { possibilidade de reúso. } \\
\text { Existem diversos REA e cada um } \\
\text { tem a sua complexidade. O } \\
\text { processo de reúso deve levar isso } \\
\text { em consideração. } \\
\text { Pouco conhecimento técnico por } \\
\text { parte dos usuários para adaptar o } \\
\text { REA para o seu contexto. }\end{array}$ & 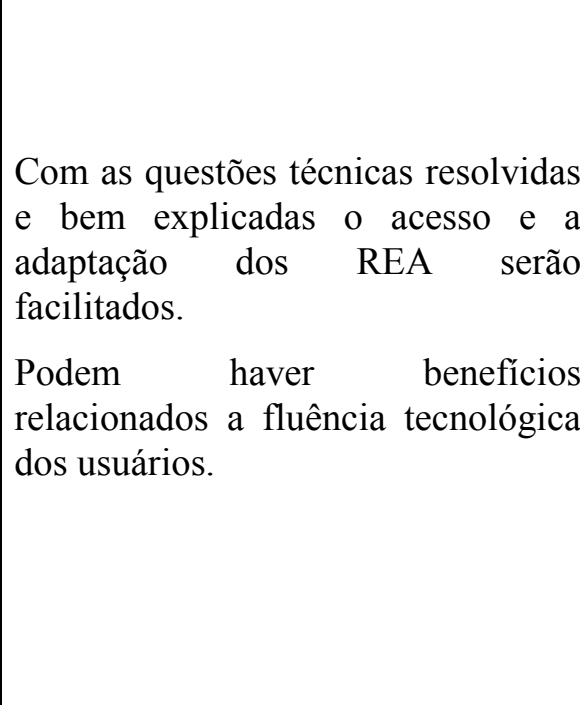 \\
\hline $\begin{array}{l}\text { Evidências de } \\
\text { inclusão }\end{array}$ & $\begin{array}{l}\text { Muitas barreiras (técnicas, } \\
\text { culturais, linguísticas, entre outras) } \\
\text { impedem que as pessoas tenham } \\
\text { acesso aos REA. } \\
\text { Há pouca discussão quanto a como } \\
\text { projetar REA multiculturais. }\end{array}$ & $\begin{array}{l}\text { Eliminar essas barreiras significa } \\
\text { oferecer uma educação mais aberta e } \\
\text { inclusiva. }\end{array}$ \\
\hline
\end{tabular}




\begin{tabular}{|c|c|c|}
\hline $\begin{array}{l}\text { Melhorias na } \\
\text { percepção da } \\
\text { comunidade }\end{array}$ & $\begin{array}{l}\text { Alunos e professores estão pouco } \\
\text { conscientes do que é um REA. } \\
\text { A produção de REA ainda está } \\
\text { concentrada em instituições, mesmo } \\
\text { assim, o seu uso no processo de } \\
\text { ensino-aprendizagem ainda é } \\
\text { tímido. }\end{array}$ & $\begin{array}{l}\text { Com REA de fato abertos e } \\
\text { flexíveis, a compreensão dos } \\
\text { benefícios e a percepção para as } \\
\text { vantagens dessa solução irão } \\
\text { melhorar. }\end{array}$ \\
\hline $\begin{array}{l}\text { Padrões } \\
\text { abertos }\end{array}$ & $\begin{array}{l}\text { Apesar da existência de muitos } \\
\text { padrões, os repositórios de REA } \\
\text { ainda apresentam problemas de } \\
\text { compatibilidade. } \\
\text { REA existem em uma enorme } \\
\text { variedade de formatos técnicos. } \\
\text { Faltam de ferramentas abertas } \\
\text { específicas para a mudança e remix } \\
\text { REA. }\end{array}$ & $\begin{array}{l}\text { Com licenças abertas os REA se } \\
\text { tornam mais adequados para o reúso } \\
\text { e compartilhamento. } \\
\text { Com os padrões abertos aplicados } \\
\text { nos repositórios, a localização, o } \\
\text { reúso e o compartilhamento ficam } \\
\text { mais fáceis. }\end{array}$ \\
\hline
\end{tabular}

A primeira oportunidade mostrada na Tabela 1 - Definição de processos de design instrucional - coloca em foco o tópico design instrucional. A motivação para esta oportunidade reside no fato de que sabemos que existem questões multiculturais a serem levadas em consideradas, no entanto, faltam iniciativas de como considerar essas questões nos REA. Além disso, é necessário pensar de forma mais ampla e considerar essas questões no design e não somente em fases ou partes isoladas.

A segunda oportunidade, "Evidências de reúso", chama a atenção para a necessidade de mais iniciativas e estudos acerca do reúso de REA. Silveira (2016) aponta alguns trabalhos que mostram as dificuldades durante esse processo nada trivial, como defendem algumas pesquisas.

A terceira oportunidade torna explícita a preocupação com as questões multiculturais. Na Seção 2 deste trabalho, vimos que há vários aspectos a serem levados em consideração quando falamos de multiculturalidade (etnia, idioma, gênero, religião, valores morais, etc.). $\mathrm{O}$ aspecto do idioma traz várias oportunidades de pesquisa no âmbito dos REA, tendo em vista que a grande maioria, 48\% deles são disponibilizados em língua inglesa e baseados na cultura ocidental, conforme aponta Nie (2013). Não é difícil imaginar que isto torna, muitas vezes, difícil o reúso em outros países que não possuem a mesma cultura e idioma.

A quarta oportunidade elencada neste trabalho aponta para soluções que busquem evidenciar os REA multiculturais, não só mostrando que eles existem, mas deixando explícito que eles podem ser considerados como verdadeiras alternativas aos materiais educacionais usados atualmente.

$\mathrm{Na}$ quinta oportunidade (Padrões abertos), podemos encontrar implicações em todas as outras oportunidades aqui apresentadas. Por exemplo, os padrões abertos favorecem todo o processo de design e reúso de REA, pois promovem melhorias desde a elaboração de conteúdos até a comunicação entre pessoas e instituições, além de promover a interoperalibidade entre plataformas, repositórios e tecnologias. 
Por fim, são elencadas algumas ações para medir o sucesso alcançados na busca por soluções para este desafio, bem como ações a serem tomadas nos próximos dez anos:

\section{Ações para avaliar o sucesso alcançado}

- Acompanhar as produções técnicas e científicas sobre o desafio;

- Verificar a satisfação de alunos de cursos baseados em REA multiculturais;

- Analisar a satisfação e as opiniões de profissionais e instituições que produzem e ofertam cursos com REA;

- Analisar e mensurar a qualidade de REA multiculturais.

\section{Ações a serem tomadas na próxima década}

- Incentivar à criação de REA multiculturais;

- Fomentar a criação de disciplinas voltadas para essa temática em cursos de graduação e pós-graduação na área de Informática na Educação;

- Fortalecer e formar grupos de pesquisa na área;

- Incentivar o trabalho interdisciplinar e interinstitucional, na produção de REA multiculturais e na produção científica sobre esse desafio.

- Criar workshops sobre o tema em eventos nacionais e internacionais.

- Organizar repositórios e construir plataformas que possam apoiar o desenvolvimento de REA multiculturais.

- Sensibilizar agentes públicos sobre o tema e as vantagens e benefícios de uma educação mais aberta e inclusiva.

\section{Conclusões}

No cenário atual se tem discutido como vencer as várias barreiras que impedem o acesso ao conhecimento e como, diante dos avanços das TIC, repensar as práticas e o papel das instituições de ensino. Nesse cenário, a preocupação em vencer as barreiras dos aspectos relacionados à multiculturalidade e a criação de recursos educacionais que promovam a disseminação do conhecimento é uma tendência. Por isso, é importante tratar os aspectos da multiculturalidade no design de recursos educacionais abertos, pois influenciam na forma como um REA é entendido, projetado, desenvolvido e utilizado. Assim, este trabalho buscou traçar um panorama de pesquisa e desenvolvimento para a década seguinte, esperando contribuir com as discussões no âmbito da Informática na Educação.

\section{Referências}

AMIEL, T.; SQUIRES, J.; OREY, M. (2009). Four strategies for designing instruction for diverse cultures. Educational Technology, v. 49, n. 6, p. 28-34, 2009. Knuth, D. E. (1984), The TeXbook, Addison Wesley, $15^{\text {th }}$ ed.

AMIEL, T.; OREY, M.; WEST, R.. (2011) Recursos Educacionais Abertos: modelos para localização e adaptação. ETD - Educ. Tem. Dig., Campinas, v.12, p.112-125.

BANZATO, M. (2012). Barriers to teacher educators seeking, creating and sharing open educational resources: An empirical study of the use of OER in education in Italy. In: 15th ICL, Villach: IEEE, 2012. 
BARANAUSKAS, M.C.C.; de SOUZA, C.S.; PEREIRA, R. (2011) I GranDIHC-BR Grandes Desafios de Pesquisa em Interação Humano-Computador no Brasil 2012 2022. Relatório Técnico. Disponível em: http://comissoes.sbc.org.br/ceihc/documentos/RT_GranDIHC_BR_2012.pdf. Acesso em: 14 de Abril de 2017.

CANEN, A. (2008). O multiculturalismo e o papel da pesquisa na formação docente: uma experiência de currículo em ação. Currículo sem Fronteiras, v.8, p.17-30, 2008.

CHANDRAMOULI, K.; STEWART, C.; BRAILSFORD, T.; IZQUIERDO, E. (2008). CAE-L: An Ontology Modelling Cultural Behaviour in Adaptive Education. In: Third International Workshop on Semantic Media Adaptation and Personalization, [S.1], 2008. Proceedings... IEEE Computer Society, 2008, p. 183- 188.

CHEN, Qing. (2010). Use of Open Educational Resources: Challenges and Strategies. In: TSANG, P., et al (Org.). Hybrid Learning, v.6248, 2010. p. 339-351. LNCS

GASPARINI, I. (2013). Aspectos Culturais no Modelo do Usuário em Sistemas Adaptativos Educacionais: Fundamentos, Proposta e Experimentação. Tese (Doutorado em Computação). Instituto de Informática - UFRGS, 2013.

HATAKKA, M. (2009): Build it and they will come? Inhibiting factors for re-use of open content in developing countries. The Electronic Journal of Information Systems in Developing Countries, 2009.

LOM (2000). LOM working draft v4.1. Disponível em: http://ltsc.ieee.org/doc/wg12/LOMv4.1.htm . Acesso em: 14 de abril de 2017.

MUÑOZ-ARTEAGA, J., VANDERDONCKT, J., GONZÁLEZ-CALLEROS, J. M., Orey, M. (2011). An Architectural Model for Designing Multicultural Learning Objects. In ACHI 2011, pp. 249 - 253. Gosier, Guadeloupe, France: IARIA.

NIE, M. (2013). Report on Comparative Analysis of Transversal OER Initiatives. [S. I.]: POERUP Policies for OER Uptake, 2013. 44 p.

OECD (2007).Giving knowledge for free: the emergence of open educational resources. OCDE Publishing. Disponível em: http://www.oecd.org/edu/ceri/38654317.pdf Acesso em: 01 abril 2017.

OKADA, A.; OKADA, S. (2007). Novos Paradigmas na Educação Online com a Aprendizagem Aberta. In: Anais da V Conferência Internacional de TIC Educação, Braga, 2007.

SBC (2006). Grandes Desafios da Pesquisa em Computação no Brasil - 2006 - 2016. Relatório Técnico. Disponível em: https://www.gta.ufrj.br/rebu/arquivos/SBCGrandes.pdf . Acesso em: 12 de maio de 2017.

SILVEIRA, I. F. (2016). OER and MOOC: The need for openness. Issues in Informing Science and Information Technology, vol. 13, p. 209-223, 2016.

UNESCO. (2002). Forum on the impact of open courseware for higher education in developing countries: Final report. Disponível em: www.unesco.org/iiep/eng/focus/opensrc/PDF/OERForumFinalReport.pdf . Acesso em: 12 de maio de 2017. 